\title{
ESTADO DEL CONOCIMIENTO SOBRE EL CABRITO DEL FRAMBUESO (CF), AEGORHINUS SUPERCILIOSUS (GUÉRIN) (COLEOPTERA: CURCULIONIDAE)
}

\author{
KNOWLEDGE OF THE “CABRITO DEL FRAMBUESO” WEEVIL (CF) \\ AEGORHINUS SUPERCILIOSUS (GUÉRIN) (COLEOPTERA: \\ CURCULIONIDAE)
}

\author{
Leonardo Parra B. ${ }^{1}$; Ana Mutis T. ${ }^{1}$; Alfonso Aguilera $P^{2}$; \\ Ramón Rebolledo R. ${ }^{2}$; Andrés Quiroz $C^{3}$
}

\begin{abstract}
RESUMEN
El cabrito del frambueso, Aegorhinus superciliosus, es una plaga importante en la zona centro sur de Chile. Afecta principalmente al complejo de frutales menores denominados berries, tales como zarzaparrilla, frutilla, grosellero, frambuesa y arándano. El estado larval se alimenta a nivel de cuello y raíces, construyendo galerías superficiales, pudiendo provocar la muerte de la planta atacada. Los adultos ocasionan daños a brotes y frutos. Pese a la importancia que representa este insecto desde el punto de vista productivo y económico, existe escasa información referente a su biología. Por lo tanto, el objetivo de esta revisión es entregar antecedentes sobre el conocimiento de la biología y control de A. superciliosus.
\end{abstract}

Palabras clave: Aegorhinus superciliosus, Curculionidae, hospedero, ontogenia, etología, control.

\section{ABSTRACT}

The weevil Aegorhinus superciliosus is a serious pest in the south of Chile, affecting mainly the complex denominated berries, such as currant, strawberry, gooseberry, raspberry and blueberry. The larvae feeds on neck and roots, building superficial galleries, causing the death of the plant. In spite of the economic importance of this insect, there is not to much information referring to its biology and control. Therefore, the objective of this review is to give antecedent on the knowledge and its advance on biology and control of A. superciliosus.

Key words: Aegorhinus superciliosus, Curculionidae, host, ontogeny, ethology, control.

\section{INTRODUCCIÓN}

La fruticultura en Chile ha experimentado un fuerte desarrollo desde las últimas décadas del siglo pasado. Esto se ha expresado tanto en un incremento en el área cultivada (ODEPA, 2005) como en la diversidad de las áreas geográficas en las cuales estos cultivos se están realizando. Esta situación ha permitido que numerosas especies de insectos, tanto nativas como introducidas, hayan colonizado estos frutales, transformándose algunas de ellas en plagas económicamente importantes para la agricultura del sur de Chile (Carrillo, 1993).

Chile se ha convertido en el mayor exportador de fruta de Sudamérica hacia los países del hemisferio norte, debido a que su condición climática permite el buen crecimiento de la fruta y al hecho de que la cosecha se lleve a cabo en una estación del año opuesta al hemisferio norte y por lo tanto no se encuentra con fruta fresca propia en los mercados invernales de EE.UU. y Europa (Muñoz et al., 1989).

\footnotetext{
Universidad de La Frontera. Temuco, Chile. E-mail: 1parra@ufro.cl

Laboratorio de Entomología, Universidad de La Frontera. Temuco, Chile.

Departamento de Ciencias Químicas, Universidad de La Frontera. Temuco, Chile. E-mail: aquiroz@ufro.cl
} 
Al ser los frutales cultivos permanentes, pasan a constituir un agroecosistema nuevo, lo que sumado al incremento de la superficie, como es el caso del arándano, origina un cambio de hábitat favorable para especies de insectos, especialmente nativos, que antes tenían como únicos hospederos árboles y plantas autóctonas. Ellos pueden alimentarse y remover partes de una planta o interferir con el desarrollo de otros órganos de esa planta, tal como ocurre con algunos gorgojos que intervienen negativamente en el desarrollo de algunos berries al atacar diferentes partes de la planta (Himelrick \& Galletta, 1990). Este es el caso del cabrito del frambueso (CF), Aegorhinus superciliosus (Coleoptera: Curculionidae), insecto nativo del cono sur de América (Chile y Argentina), cuya larva posee poderosas mandíbulas con las cuales corta y tritura las raíces y raicillas de la planta, horadando finalmente la raíz principal cerca del cuello donde construye una cámara para pupar. Toda esta actividad larvaria provoca un detrimento en la planta, que puede derivar en su muerte (Kuschel 1951; Aguilera, 1988, 1995; Carrillo, 1993, Elgueta, 1993). Debido a que este ataque es producido subterráneamente, el control químico directo con insecticidas es prácticamente ineficaz. Otra limitante que presenta este tipo de control, cuando es dirigido al estado adulto, es el largo efecto residual y periodo de carencia de los productos utilizados, los cuales al ser aplicados cercanos al periodo de floración son nocivos para las abejas polinizantes (Aguilera, 1995).

Pese a la importancia que representa este insecto desde el punto de vista productivo y económico, existe escasa información referente a su biología, limitándose principalmente a los aportes de Aguilera (1988, 1995), Aguilera \& Rebolledo (2001), Reyes (1993) y Pérez (1994). Por lo tanto, el objetivo de esta revisión es entregar antecedentes sobre el conocimiento y su avance sobre la biología y control del CF.

\section{ANTECEDENTES GENERALES}

Los Aterpini americanos (Coleoptera: Curculionidae) se han clasificado generalmente en tres géneros: Aegorhinus Erichson, Alastoropolus Kuschel y Micropolus Kuschel (Kuschel 1952; Morrone \& Roig-Juñent 1999). Dentro de esta tribu, Aegorhinus es el género más diversificado, con 24 especies (Kuschel 1951, 1952, 1955; Cekalovic
1970; Elgueta 1974, 2000), el cual se caracteriza por tener especies de diferentes formas, recibiendo los adultos nombres tales como caballitos, cabritos y otros (Peña 1986).

Según Aguilera (1992), la especie Aegorhinus superciliosus (Guérin) puede ser denominada como falso gusano de la frambuesa, marinerito de la frambuesa, burrito de la frambuesa o cabrito del frambueso. De acuerdo al nombre genérico, la denominación vulgar más adecuada que se propone es "cabrito de la frambuesa" (Aego: cabro; rhinus: nariz). Con respecto al nombre científico, Kuschel (1951) señala que desde su descripción original han surgido otras, dando una sinonimia de 30 nombres. Según Elgueta (1993), A. superciliosus presenta una gran variedad morfológica y por no existir barreras geográficas que delimiten la distribución de ellas, las subespecies reconocidas por Kuschel (1951) y las citadas por Prado (1991) deben ser consideradas como morfos.

\section{DISTRIBUCIÓN GEOGRÁFICA}

El CF se encuentra distribuido en el cono sur de América. En Chile se ubica entre la VII a X Regiones (Elgueta, 1993). Sin embargo, otros autores discrepan sobre su distribución debido a que se refieren a otras razas de A. superciliosus (Aguilera, 1988; Artigas, 1994; Arias, 2000). En Argentina, es citado para la provincia de Neuquén (Aguilera, 1995).

\section{DESCRIPCIÓN MORFOLÓGICA}

La descripción morfológica de los diferentes estados del CF se basa principalmente en lo informado por Kuschel (1951) y a los aportes realizados por Reyes (1993) y Pérez (1994).

\section{DESCRIPCIÓN DEL ESTADO DE HUEVO}

Según Reyes (1993), los huevos del CF (Fig. 1) se caracterizan por ser de forma ovoide, color amarillo pálido y no presentar ornamentación alguna, tal como sucede con la mayoría de los huevos del orden Coleoptera (Britton, 1970). Estos huevos son ovipuestos en forma individual y cubiertos por un material viscoso producido por la hembra (Carrillo et al., 2002), a la vez son de consistencia blanda 


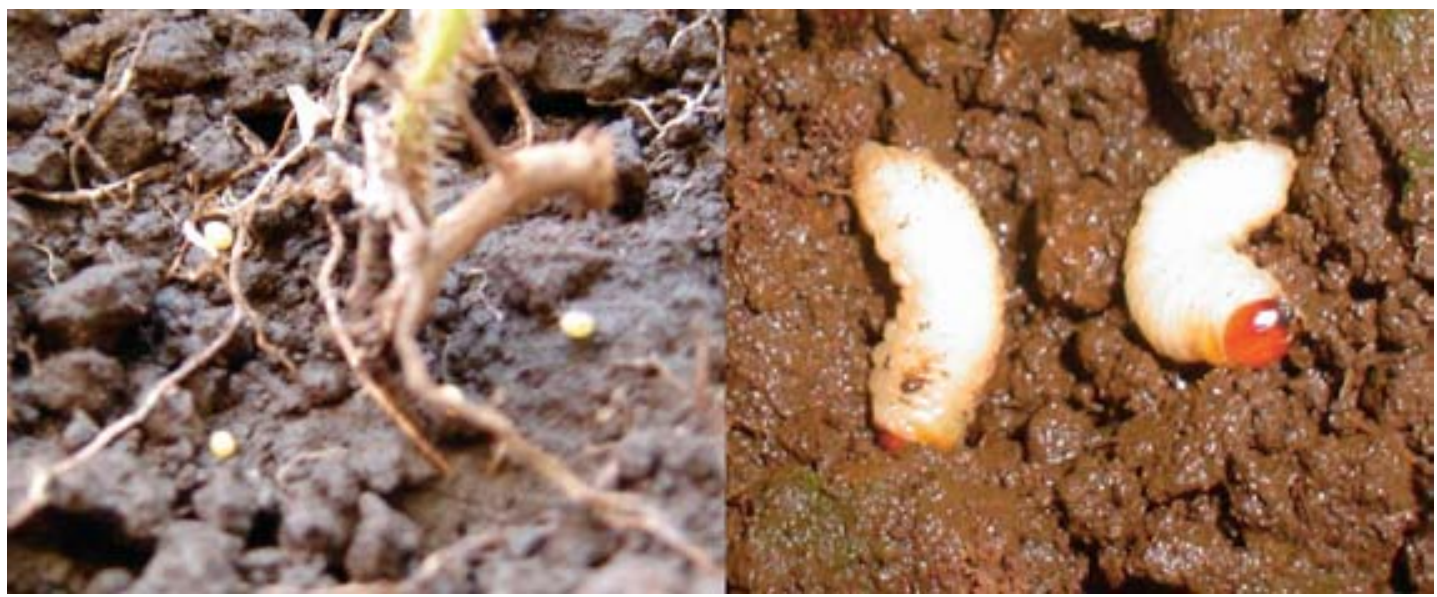

Figura 1. Huevos y larvas de Aegorhinus superciliosus.

y de extrema fragilidad (Reyes, 1993). El tamaño de los huevos de A. superciliosus es pequeño, con un diámetro ecuatorial promedio de 1,23 $\pm 0,0985$ $\mathrm{mm}$ y un diámetro polar promedio de 1,55 $\pm 0,103$ mm (Reyes, 1993).

\section{DESCRIPCIÓN DEL ESTADO DE LARVA}

Reyes (1993) indica que esta es ápoda, de color blanco amarillento, cuya cabeza está en posición hipognata, libre, esclerosada y no posee ocelos, pero sí unas poderosas mandíbulas con las cuales corta y tritura las raicillas y raíces de su hospedero (Aguilera, 1995) (Fig. 1). Presenta tres segmentos torácicos y nueve segmentos abdominales, los que con excepción del noveno poseen un par de espiráculos distribuidos en línea curva a cada lado del abdomen. La larva recién emergida del huevo mide aproximadamente $1,5 \mathrm{~mm}$ y la plenamente desarrollada alrededor de $2 \mathrm{~cm}$, tamaño que alcanza a través de sucesivos cambios de muda (Aguilera, 1988). Aguilera \& Rebolledo (2001) determinaron que la larva de $A$. superciliosus, en condiciones de laboratorio, se desarrolla a través de 14 estadios, en un periodo variable entre 313 y 487 días, con promedio de 435,01 $\pm 15,23$ días.

\section{DESCRIPCIÓN DEL ESTADO DE PUPA}

Pérez (1994) informa que las pupas de esta especie son robustas, de color blanco crema y se encuentran enterradas en el suelo, en celdillas que construye la larva al completar su desarrollo al interior de las raíces (Fig. 2). El cuerpo se encuentra provisto de setas robustas de color café oscuras, cortas, con forma de aguja y montadas en tubérculos. En investigaciones realizadas por este mismo autor, se determinó que estas pupas alcanzan un largo de 15,25 $\pm 0,07 \mathrm{~mm}$ y un ancho máximo de $7,73 \pm 0,46 \mathrm{~mm}$.

\section{DESCRIPCIÓN DEL ESTADO ADULTO}

Tanto las hembras como los machos alcanzan entre 15 a $20 \mathrm{~mm}$ de longitud (Aguilera, 1988; Cisternas et al., 2000), con cuerpo alongado y de lados subparalelos (Marvaldi \& Lanteri, 2005) (Fig. 3). La cabeza con antenas de diez segmentos y ojos finamente facetados. El rostrum se dirige hacia abajo y no hacia a delante, como en otros curculiónidos. El tórax más largo que ancho con escamas blancas esparcidas en la superficie. Los élitros presentan siete bandas o filamentos blancos transversales a su largo, en algunos ejemplares más notorios que otros (Aguilera, 1988; Cisternas et al., 2000). El dorso del cuerpo es de color gris obscuro a negro, rugoso y esclerotizado. Sus patas son tan largas como la mitad del largo del cuerpo, destacándose en ellas notoriamente los tarsos, con una fórmula tarsal aparente 4-4-4, con el último tarsito alargado provisto de dos uñas terminales (Aguilera, 1988).

Esta especie presenta algunos caracteres que permiten diferenciar machos de hembras. Kuschel (1951) señala que el macho es de tamaño menor y más angosto que la hembra. Las tibias delanteras se presentan casi siempre más arqueadas en los machos que en las hembras. Al respecto, Reyes (1993) señala que el fémur del tercer par de patas 


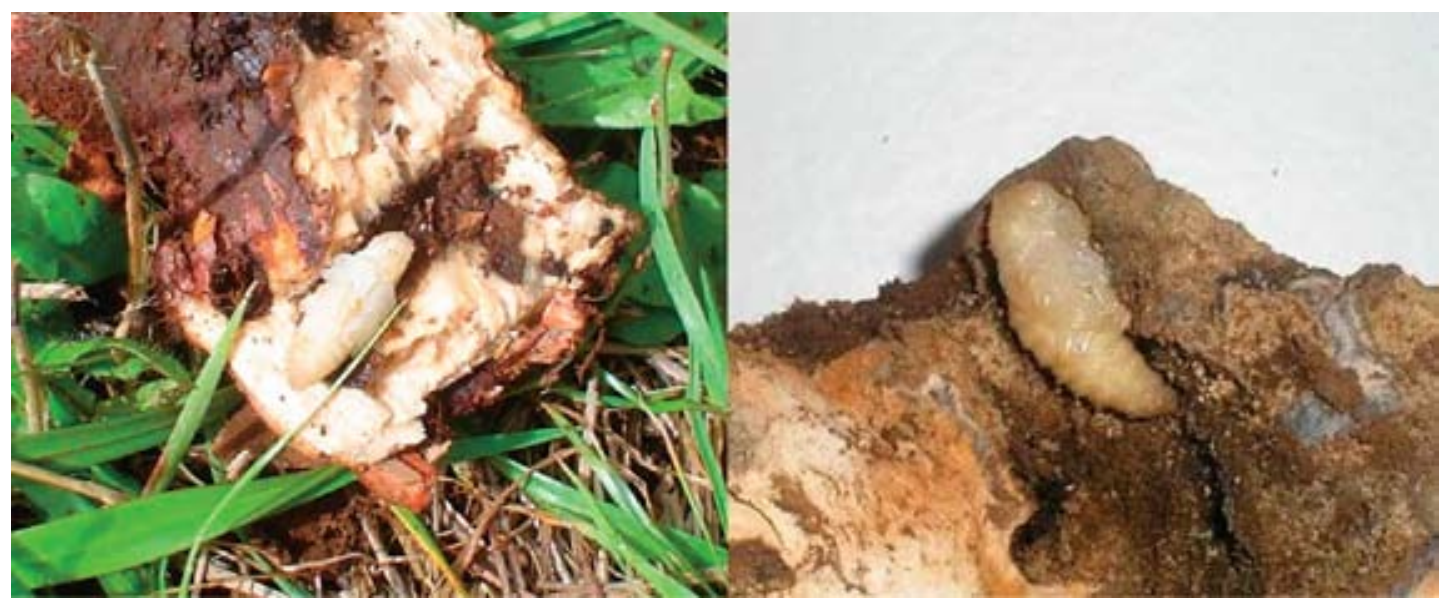

Figura 2. Pupas de Aegorhinus superciliosus.

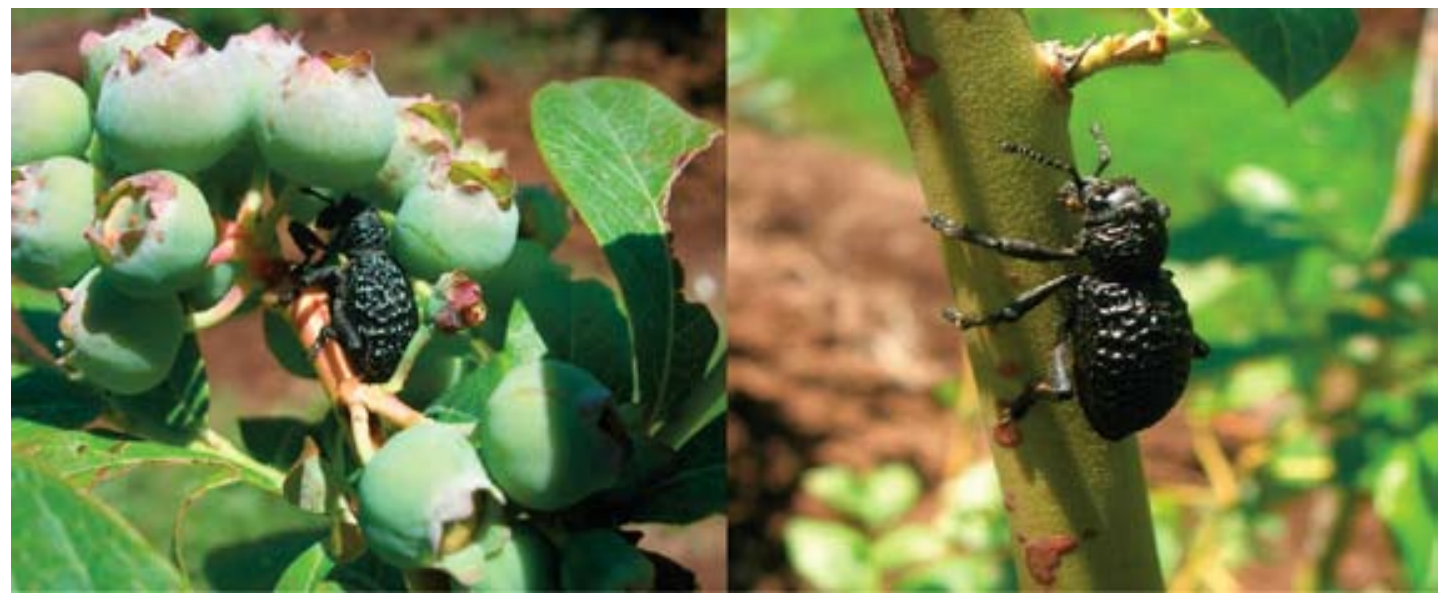

Figura 3. Adultos de Aegorhinus superciliosus.

sobrepasa la sutura posterior del cuarto esternito en los machos, mientras que en las hembras alcanza el cuarto esternito, pero no sobrepasa la sutura.

\section{ONTOGENIA Y ETOLOGÍA DEL CF}

\section{CICLO VITAL}

Según Aguilera (1988), la primera aproximación al ciclo vital del CF, observado en la IX Región de La Araucanía, durante dos temporadas (1986-1987), indica que este insecto presenta al menos una generación anual, pasando el invierno preferentemente en estado larvario, aunque se han encontrado adultos vivos entre terrones de suelo durante el mes de julio, lo que indica que también pueden invernar en dicho estado. Sin embargo, cabe señalar que la larva se encuentra activa durante todo su desarrollo. Pese a contar con estos antecedentes, Elgueta (1993) indica que el ciclo es desconocido, encontrándose los adultos entre los meses de octubre a junio. Esto es coincidente con lo señalado por Carrillo (1993), quien afirma que los adultos emergen generalmente en noviembre y diciembre, siendo su vida como adulto de aproximadamente seis meses y caracterizándose por ser altamente fecundos.

\section{HOSPEDEROS}

Hacia los años 50, esta especie fue citada por Kuschel (1951) atacando diversos árboles frutales, 
donde estos insectos suben durante el día hacia la fronda para alimentarse del follaje tierno y bajan hacia la parte basal al atardecer, permaneciendo en ese sector. El desarrollo larval de esta especie se efectúa a nivel de cuello y al interior de raíces de las plantas hospederas, ya sean nativas o introducidas (Kuschel, 1951, 1952; Elgueta \& Arriagada, 1989; Elgueta, 1993, 2000). Estos hospederos y su evolución hasta la actualidad son mostrados en la tabla 1. El CF también ha sido citado como elemento habitual de la selva valdiviana junto a $A$. nodipennis (Hope) (Solervicens \& Elgueta, 1994).

\section{DAÑO OCASIONADO POR EL CF}

La hembra adulta ovipone en el cuello de su hospedero o en la superficie del suelo (Caballero 1972), en áreas circundantes a la planta, recubriendo los huevos con una sustancia mucilaginosa, la que proporcionaría una defensa contra la desecación y al mismo tiempo actuaría desincentivando la depredación (Pérez 1994; Cisternas et al. 2000). Al nacer, las larvas se trasladan al interior del suelo, hacia la zona radicular de plantas sanas, cerca de la raíz principal. $\mathrm{Al}$ introducirse a la raíz, horadan

Tabla 1

Lista de hospederos de A. superciliosus

\begin{tabular}{|c|c|c|c|}
\hline Nombre vulgar & Nombre científico & Familia & Referencia \\
\hline \multicolumn{4}{|l|}{ Nativos } \\
\hline $\begin{array}{l}\text { Chilca, verbena } \\
\text { de tres esquinas }\end{array}$ & $\begin{array}{l}\text { Baccharis racemosa } \\
\text { (R. et. P) D.C. }\end{array}$ & Asteraceae & Morrone \& Roig-Juñent, 1999. \\
\hline Canelo & Drimys winteri J. R. et Forster & Winteraceae & Kuschel, 1951; Morrone \& Roig-Juñent, 1999. \\
\hline Zarzaparrilla & $\begin{array}{l}\text { Ribes nigrum L., Ribes } \\
\text { rubrum } L .\end{array}$ & Saxifragaceae & $\begin{array}{l}\text { Aguilera, 1988; Guerrero \& Aguilera, 1989; Aguilera, } \\
\text { 1990; Prado, 1991; Carrillo, 1993; Elgueta, } 1993 .\end{array}$ \\
\hline Maitén & Maytenus boaria Mol. & Celastraceae & $\begin{array}{l}\text { Aguilera, 1988; Guerrero \& Aguilera, 1989; Arias, } \\
\text { 2000; Sandoval, } 2007 .\end{array}$ \\
\hline Coigüie & Nothofagus dombeyi (Mirb.) & Nothofagaceae & Arias, 2000; Klein \& Waterhouse, 2000. \\
\hline \multicolumn{4}{|l|}{ Introducidos } \\
\hline Manzano & Malus domestica Borkh & Rosaceae & $\begin{array}{l}\text { Kuschel, 1951; Prado, 1991; Guerrero, 1992; Elgueta, } \\
\text { 1993; Klein \& Waterhouse, } 2000 .\end{array}$ \\
\hline Membrillo & Cydonia oblonga Miller & Rosaceae & Kuschel, 1951. \\
\hline Frambueso & Rubus idaeus L. & Rosaceae & $\begin{array}{l}\text { Aguilera, 1988; Guerrero \& Aguilera, 1989; Aguilera, } \\
\text { 1990; 1994; Prado, 1991; Carrillo, 1993; Elgueta, } 1993 .\end{array}$ \\
\hline Frutilla & Fragaria ananassa Duch & Rosaceae & $\begin{array}{l}\text { Aguilera, 1988; Guerrero \& Aguilera, 1989; Aguilera, } \\
\text { 1990; Prado, 1991; Carrillo, 1993; Elgueta, } 1993 .\end{array}$ \\
\hline Mora & Rubus constrictus Muell. et Lef. & Rosaceae & $\begin{array}{l}\text { Aguilera, 1988; Guerrero \& Aguilera, 1989; Aguilera, } \\
\text { 1990; Prado, 1991; Carrillo, 1993; Elgueta, } 1993 .\end{array}$ \\
\hline Arándano & Vaccinium corymbosum $\mathrm{L}$. & Ericaceae & $\begin{array}{l}\text { Aguilera, 1988; Guerrero \& Aguilera, 1989; Aguilera, } \\
\text { 1990; Prado, 1991; Carrillo, 1993; Elgueta, } 1993 .\end{array}$ \\
\hline Romaza & Rumex crispus $\mathrm{L}$. & Poligonaceae & Kuschel, 1951; Morrone \& Roig-Juñent, 1999. \\
\hline Grosellero & Ribes grossularia & Saxifragaceae & $\begin{array}{l}\text { Aguilera, 1988; Guerrero \& Aguilera, 1989; Aguilera, } \\
\text { 1990; Prado, 1991; Carrillo, 1993; Elgueta, } 1993 .\end{array}$ \\
\hline Sauce & Salix viminalis $L$. & Salicaceae & Aguilera, 1988; Elgueta, 1993. \\
\hline $\begin{array}{l}\text { Avellano } \\
\text { europeo }\end{array}$ & Corylus avellana $L$. & Betulaceae & $\begin{array}{l}\text { Aguilera, 1988, 1995; Guerrero \& Aguilera, 1989; } \\
\text { Grau et al., } 2001 .\end{array}$ \\
\hline Ciruelo & Prunus salicina Lindl. & Rosaceae & Rivera, 1905; Kuschel, 1951; Prado, 1991. \\
\hline
\end{tabular}


una galería cuya entrada cubren o tapan con aserrín y deposiciones. Esta galería llega hasta el cuello de la planta, donde la larva pupa para terminar su desarrollo. Esta acción puede ocasionar la muerte de la planta atacada (Kuschel, 1951; Elgueta, 1993; Cisternas et al., 2000). Los adultos ocasionan daños a brotes y frutos. En ataques graves la planta sufre una severa defoliación y en algunos casos la muerte (Carrillo, 1993). En observaciones realizadas sobre la distribución del daño en la planta causado por adultos, se encontró que éste generalmente se concentra en la parte alta de la planta, lo cual puede ser efecto de un geotropismo negativo, o bien esta concentración del consumo en la región apical se debe a la calidad del recurso alimenticio que está constituido por tejido más blando y nutritivo (Pérez, 1994).

El daño producido por esta especie había sido informado por Rivera (1905) en la "Actes de la Société Scientifique du Chili", donde informó sobre los daños provocados por Lophotus superciliosus en manzano. L. superciliosus corresponde actualmente, según lo reportado por Blackwelder (1957) y Elgueta \& Jackson (1987), a la especie Aegorhinus superciliosus.

A pesar de su importancia, los antecedentes sobre su biología se refieren sólo a algunos aspectos de sus hábitos de vida y desarrollo (Reyes, 1993; Pérez, 1994; Aguilera, 1995; Aguilera \& Rebolledo, 2001).

\section{IMPORTANCIA ECONÓMICA}

La importancia económica de este insecto fue establecida el año 1988, cuando Aguilera cita al CF como el insecto plaga de mayor importancia económica en plantaciones de arándanos por los daños que provoca. Luego, González (1989) resume su importancia económica como ocasional en troncos y ramas de frambueso o en la raíz del ciruelo. Prado (1991) lo menciona como una plaga ocasional en arándano, frambueso, frutilla, grosellero, manzano, mora y zarzaparrilla. Por su parte, Aguilera (1990) considera a esta especie como potencialmente cuarentenaria, considerando que se encuentra solamente en el cono sur de América (zona sur de Chile y Argentina). Posteriormente, Aguilera (1995) lo vuelve a citar como una plaga de importancia económica, debido al aumento de su población, lo que se asocia al incremento en las plantaciones de arándano en el país. Durante 1996, el Servicio Agrícola y Ganadero (SAG) modificó la Resolución No 1910 de 1982 en los puntos 3 y 4 del capítulo II, donde se especifica que los criaderos, viveros y depósitos deberán expender plantas libres de plagas especialmente las señaladas en sus nóminas. Entre éstas se nombra a Aegorhinus sp. como plaga en frutales menores, específicamente berries. La constatación de éstas y otras plagas habilita al SAG para que, a través de sus inspectores, se dispongan los tratamientos necesarios o la eliminación de las plantas afectadas. Finalmente, la Comunidad Europea (2003) a través del Diario Oficial de la Unión Europea considera a las especies A. phaleratus y A. superciliosus como organismos nocivos para las exportaciones chilenas a países que corresponden a dicha comunidad.

\section{CONTROL DEL CF}

En Chile, la principal estrategia de control del CF consiste en la utilización de productos químicos; sin embargo, a pesar de lograr cierta efectividad en adultos, ha debido complementarse con otros métodos de control, sin que se hayan obtenido resultados óptimos. Dentro de los métodos utilizados se mencionan el control fisicomecánico, químico, cultural y biológico. Además se debe considerar en cierta medida el control natural.

\section{CONTROL NATURAL}

Ripa y Caltagirone (1994) lo definen como aquel control biológico que ocurre en forma natural, sin intervención del hombre, siendo los enemigos naturales importantes en la reducción del organismo nocivo. En el suelo actúa un complejo de patógenos (bacterias y hongos), nematodos e insectos que actúan sobre larvas y pupas de Aegorhinus. Prado (1991) señala al himenóptero bracónido del género Centistes y al hongo entomopatógeno Metarhizium anisopliae como enemigos naturales de este insecto en estado larvario (Aguilera, 1994). Por su parte, Carrillo (1993) menciona que este insecto presenta escasos enemigos naturales, no obstante, se ha detectado la presencia de larvas de taquínidos (Diptera) en adultos.

\section{CONTROL ARTIFICIAL}

\section{Control fisicomecánico}

La práctica de este método constituye una alternativa selectiva de control cuando la densidad 
poblacional del insecto no representa un peligro inminente. Este método consiste en la colecta manual y periódica, con la posterior incineración de los adultos del CF (Aguilera, 1995).

\section{Control químico}

Este método de control, dirigido al estado larvario del insecto, es prácticamente ineficaz cuando éste se encuentra ya instalado subterráneamente en la planta, de tal manera que la estrategia de control químico en huertos establecidos donde no se han tomado medidas preventivas debe ser dirigida al estado adulto (Aguilera, 1995).

Se ha determinado que los insecticidas organofosforados no selectivos, azinfos etil y azinfos metil, son los productos más efectivos en el control de adultos en condiciones semicontroladas (Aguilera, 1988, 1995). Carrillo (1993) recomienda la utilización de insecticidas de la familia de los carbamatos como es el caso de carbofurán. Sin embargo, estos productos presentan un periodo de carencia de al menos 30 días, siendo esta condición una limitante según el manejo que se requiera para las diversas especies frutales, tal como el arándano. Otra limitante, de acuerdo al manejo del azinfos metil y azinfos etil, es el largo efecto residual que presentan estos insecticidas, los cuales al ser aplicados cercanos al periodo de floración son nocivos para los agentes polinizadores (Aguilera, 1995).

Dado el inconveniente que presentan los insecticidas de amplio espectro, se ha experimentado con productos que inhiben la formación de quitina. Inicialmente se probó triflumuron, aplicado directamente sobre huevos del CF, obteniéndose entre un $95-100 \%$ de efectividad según sea la dosis. No obstante, este producto no tiene acción de control sobre los adultos (Aguilera, 1995). Los primeros ensayos con triflumuron sobre el CF motivaron la realización de experimentos de campo con otros reguladores de crecimiento, con el objeto de inhibir su desarrollo embrionario. Estos productos fueron fluflenoxuron y diflumenzuron, sin embargo, estos no superaron el $70 \%$ de efectividad como promedio de dos temporadas de evaluación (Soler, 1994; Aguilera, 1995).

\section{Control cultural}

Debe ser realizado en forma preventiva, evitando la infestación de la plantación. Para ello es recomendable preparar bien el suelo a plantar y complementar con la aplicación de insecticida, si corresponde (Carrillo, 1993). Otra labor de control cultural corresponde a la utilización de cultivares tolerantes o resistentes. Se ha determinado que en arándano el cultivar Northland es el más tolerante al ataque del CF, de acuerdo a observaciones realizadas con diferentes cultivares en la provincia de Malleco (Aguilera, 1995). Con respecto a este tema, Maynet (1997) efectuó ensayos con diversos cultivares de frambueso estableciendo que el cultivar Meeker fue el menos preferido por el adulto de $A$. superciliosus, bajo condiciones semicontroladas de laboratorio.

\section{Control biológico}

Dentro de este método, destaca el uso de los hongos entomopatógenos de los géneros Metarhizium y Beauveria (Moniliales: Moniliaceae), los que se encuentran ampliamente distribuidos en el mundo y son considerados inocuos para el medio ambiente (France et al., 2000). La entrada de estos hongos al insecto se produce a través de aberturas naturales (boca, espiráculos y ano) o a través del integumento por medio de un proceso mecánico enzimático. Durante el proceso de infección el hongo produce toxinas que causan la muerte del insecto, para luego colonizar el interior del hospedante y emerger en forma de micelio al exterior, para posteriormente producir esporas (St. Legar et al., 1986; Lecuona et al., 1996; Alves, 1998). A pesar de que se ha detectado con anterioridad este tipo de hongos en Chile (Dutky, 1957; Vásquez, 1977; Guerrero et al., 1999) y se han realizado pruebas de patogenicidad, no ha existido hasta la fecha un uso expansivo de estos entomopatógenos. Los antecedentes de uso de hongos entomopatógenos en Chile muestran resultados no siempre satisfactorios, debido principalmente a que las cepas utilizadas fueron colectadas en otros países o no se realizó una selección previa con varios aislamientos (Ripa y Rodríguez, 1989).

\section{TEMAS PENDIENTES Y PROYECCIONES FUTURAS}

Debido al uso excesivo de plaguicidas sintéticos y su baja efectividad en el control del CF, el uso de atrayentes naturales de insectos surge como una valiosa alternativa a utilizar, la cual se encuentra 
inserta dentro del método de control etológico de plagas. Esto se debe a las ventajas que presenta, tales como inocuidad ecológica (alta especificidad), elevada eficiencia, no presentan problemas de resistencia, ni tampoco producen contaminación ambiental.

Durante las últimas décadas la investigación en la ecología química de los curculiónidos ha adquirido gran importancia a nivel mundial. De hecho, se ha logrado determinar la naturaleza química de compuestos de diversas plantas, denominadas kairomonas (atrayentes), con las que se han elaborado trampas atrayentes que reproducen de manera muy cercana la proporción de componentes químicos liberados por las plantas hospederas.

Sin embargo, para llevar a cabo esto y ser aplicado hacia el CF es necesario un enfoque multidimensional con una visión agroecológica del problema que constituye el control de plagas, basado en el Manejo Integrado de Plagas (MIP). Este sistema se fundamenta en la integración y aplicación estratégica de diferentes métodos de control existentes de plagas conocidas, coordinadas armónicamente dentro de una estrategia de manejo y basada en un análisis de costo/beneficio que considere los intereses e impacto en productores, sociedad y el ambiente.

\section{LITERATURA CITADA}

AGUILERA, A. 1988. Plagas del arándano en Chile. pp. 109131. En Lobos, W. (ed.). Seminario: El cultivo del arándano. INIA Carillanca (Temuco, Chile).

AGUILERA, A. 1990. Insectos fitófagos cuarentenarios asociados a frutales menores en la IX Región. IPA Carillanca (Temuco, Chile) 3: 7-11.

AGUILERA, A. 1992. Plagas subterráneas en Chile. pp. 71-105. II Reunión sobre pragas subterráneas dos paises do Cone Sul. Anais CNPMS/EMBRAPA (Minas Gerais, Brasil).

AGUILERA, A. 1994. Plagas del frambueso en la IX Región de Chile. IPA Carillanca (Temuco, Chile) 4: 23-32.

AGUILERA, A. 1995. Control selectivo de plagas en frutales de la zona sur. pp. 141-180. En Aguilera, A., Andrade, O., Díaz, J., Espinoza, N., Galdames, R. \& Norambuena, H. (eds). Seminario de Protección Vegetal. INIA Carillanca (Temuco, Chile).

AGUILERA A. \& R. REBOLLEDO. 2001. Estadios larvarios de Aegorhinus superciliosus (Guérin, 1830) (Coleoptera: Curculionidae). Rev. Chilena Ent. 28: 5-8.

ALVES, S. B. 1998. Patologia e controle microbiano: vantagens e desvantagens. pp. 21-38. En Alves, S. B. (ed.). Controle Microbiano de Insetos. Fundação de Estudos Agrários Luiz de Queiroz, Piracicaba.

ARIAS, E. 2000. Coleópteros de Chile. Fototeknika (Santiago, Chile). 209 p.
ARTIGAS, J. 1994. Entomología Económica: insectos de interés agrícola, forestal, médico y veterinario (nativos, introducidos y susceptibles de ser introducidos). Eds. Universidad de Concepción (Chile). 1126 p.

BLACKWELDER, R. E. 1957. Checklist of the Coleopterous insects of Mexico, Central America, the West Indies, and South America, part 5. Smithsonian Institution US Natural History Museum Bulletin 185. 827 p.

BRITTON, E. 1970. The insect of Australia. Melbourne University Press (Victoria). 1029 p.

CABALlERO, C. 1972. Algunos aspectos de la biología de Aegorhinus phaleratus Erichson (Coleoptera: Curculionidae), en el duraznero de Chile. Rev. Peruana Ent. 15: 186-189.

CARRILLO, R. 1993. Plagas insectiles en arbustos frutales menores. pp. 63-86. En Barriga, P. \& Neira, M. (ed.). Cultivos no tradicionales. Uniprint. (Valdivia, Chile).

CARRILLO, R.; H. PÉREZ \& M. NEIRA. 2002. Comportamiento de oviposición de Aegorhinus superciliosus (Guérin) (Coleoptera: Curculionidae). Agro Sur (Valdivia, Chile) 30: 47-50.

CEKALOVIC, T. 1970. Nueva especie para el género Aegorhinus Erichson (Coleoptera: Curculionidae). Bol. Soc. Biol. de Concepción (Chile) 42: 55-57.

CISTERNAS, E.; A. FRANCE; L. DEVOTTO \& M. GERDING. 2000. Insectos, ácaros y enfermedades asociadas a la frambuesa. Instituto de Investigaciones Agropecuarias. (Chillán, Chile). 126 p.

COMUNIDAD EUROPEA. 2003. Diario Oficial de la Unión Europea: Decisión de la Comisión de 9 de abril de 2003: C(2003) 1184. (Bruselas). 4 p.

DUTKY, S. 1957. Report on white grub control project in Chile. Agric. Téc. (Chile) 1: 92-105.

ELGUETA, M. 1974. Una nueva especie de Aterpinae (Coleoptera, Curculionidae). Rev. Chilena Ent. 8: 133-134.

ELGUETA, M. \& D. JACKSON. 1987. Nombre actual de las especies de Curculionoidea (Insecta: Coleoptera) tratadas en la obra de Gay. Rev. Chilena Ent. 15: 71-78.

ELGUETA, M. \& G. ARRIAGADA. 1989. Estado actual del conocimiento de los coleópteros de Chile (Insecta: Coleoptera). Rev. Chilena Ent. 17: 5-60.

ELGUETA, M. 1993. Las especies de Curculionoidea (Insecta: Coleoptera) de interés agrícola en Chile. Museo Nacional de Historia Natural. Publicación Ocasional (Santiago) 48: 1-79.

ELGUETA, M. 2000. Dos especies nuevas de Aegorhinus (Coleoptera: Curculionidae: Aterpini) de Chile. Acta Ent. Chilena (Santiago) 24: 7-18.

FRANCE, A.; M. GERDING; M. GERDING \& A. SANDOVAL. 2000. Patogenicidad de una colección de cepas nativas de Metarhizium spp. y Beauveria spp. en Aegorhinus superciliosus, Asynonychus cervinus y Otiorhynchus sulcatus. Agric. Téc. (Chile) 60: 205-215.

GONZÁLEZ, R. 1989. Insectos y ácaros de importancia agrícola y cuarentenaria en Chile. Editorial Ograma. (Santiago, Chile). $310 \mathrm{p}$.

GRAU, P.; A. FRANCE; M. GERDING; A. LAVIN \& A. TORRES. 2001. Preliminary evaluation of hazelnut performance in Chile. Acta Hort. 556: 49-58.

GUERRERO, J. 1992. Situación fitosanitaria del manzano en la IX Región. pp. 37-40. En Lobos, W. (ed.). Seminario: Análisis: el negocio de la manzana. Imprenta Universidad de La Frontera (Temuco, Chile). 
GUERRERO, J. \& A.AGUILERA. 1989. Plagas y enfermedades en el arándano chileno. Próxima Década (Santiago, Chile). 76: 24-29.

GUERRERO, J.; R. CARRILLO \& A. AGUILERA. 1999. Caracterización morfológica y germinación de cepas del hongo entomopatógeno Metarhizium anisopliae var. anisopliae, asociado a larvas de escarabaeidos y curculiónidos. Agro Sur (Valdivia, Chile) 27: 23-34.

HIMELRICK, D. \& GALLETTA, G. 1990. Factors that influence small fruit production. pp. 14-82. In Galletta, G. \& Himelrick, D. (eds.). Small Fruit Crop Management. Prentice Hall. (New Jersey).

KLEIN, C. \& D. F. WATERHOUSE. 2000. The distribución and importance of arthropods associated with agriculture and forestry in Chile. ACIAR Monograph 68. (Canberra, Australia). $231 \mathrm{p}$.

KUSCHEL, G. 1951. La subfamilia Aterpinae en América. Rev. Chilena Ent. 1: 205-244.

KUSCHEL, G. 1952. Los Curculionidae de la cordillera chilenoargentina ( $1^{\text {a }}$ parte). Rev. Chilena Ent. 2: 229-279.

KUSCHEL, G. 1955. Nuevas sinonimias y anotaciones sobre Curculionoidea (Coleoptera). Rev. Chilena Ent. 4: 261-312.

LECUONA, R.; B. PAPIEROK \& G. RIBA. 1996. Hongos entomopatógenos. pp 35-60. En Lecuona, R. (ed.). Microorganismos Patógenos Empleados en el Control Microbiano de Insectos Plaga. Talleres Gráficos Mariano Mas. (Buenos Aires).

MARVALDI, A. \& A. LANTERI. 2005. Key to higher taxa of South American weevils based on adult characters (Coleoptera, Curculionidae). Revista Chilena de Historia Natural 78: 65-87.

MAYNET, M. 1997. Preferencia del adulto de Aegorhinus superciliosus (Guérin) (Coleoptera: Curculionidae) frente a diferentes cultivares de frambueso y un cultivar de mora híbrida. Tesis Ingeniero Agrónomo. Universidad de La Frontera. (Temuco, Chile). 69 p.

MORRONE, J. J. \& S. ROIG-JUÑENT. 1999. Synopsis and cladistics of the American Aterpini (Coleoptera: Curculionidae, Cyclominae). Ent. Scand. 30: 417-434.

MUÑOZ, C.; W. LOBOS; A. LAVIN \& J. VALENZUELA. 1989. Potential for blueberry growing in Chile. Acta Hortic. 241: 31-37.

ODEPA. 2005. Agricultura chilena 2014: una perspectiva de mediano plazo. Ministerio de Agricultura. (Santiago, Chile). 242 p.
PEÑA, L. 1986. Introducción a los insectos de Chile. Editorial Universitaria (Santiago, Chile). 256 p.

PÉREZ, H. 1994. Descripción de aspectos morfológicos, biológicos y de comportamiento de Aegorhinus superciliosus (Coleoptera: Curculionidae). Tesis Ingeniero Agrónomo. Universidad Austral de Chile (Valdivia. Chile). 102 p.

PRADO, E. 1991. Artrópodos y sus enemigos naturales asociados a plantas cultivadas en Chile. Publicaciones Estación Experimental La Platina, Santiago. 203 p.

REYES, G. 1993. Aspectos morfológicos y biológicos de la especie Aegorhinus superciliosus (Coleoptera: Curculionidae). Tesis Ingeniero Agrónomo. Universidad Austral de Chile (Valdivia. Chile). 90 p.

RIPA, R. \& A. RODRÍGUEZ. 1989. Susceptibilidad de larvas de Naupactus xanthographus (Coleoptera: Curculionidae) a ocho aislamientos de Metarhizium anisopliae (Deuteromycotina: Hyphomycetes). Agric. Téc. (Chile) 49: 336-40 p.

RIPA, R. \& L. CALTAGIRONE. 1994. Implementación del control integrado de plagas. Rev. Frutícola (Chile). 15: 67-73.

RIVERA, M. 1905. Los insectos de las arboledas de Contulmo. Actes de la Société Scientifique du Chili. (Santiago, Chile) 15: 1-26.

SERVICIO AGRÍCOLA Y GANADERO. 1996. Departamento Protección Agrícola, Ministerio de Agricultura: Decreto Ley No 3.357 de 1980. (Santiago, Chile). 9 p.

SANDOVAL, A. 2007. Curculiónidos (Coleoptera: Curculionidae) presentes en maitén (Maytenus boaria Mol.), en el llano central de La Araucanía. Tesis Ingeniero Forestal. Universidad de La Frontera. Temuco, Chile.

SOLER, A. 1994. Efectividad de algunos reguladores de crecimiento en la inhibición de la embriogénesis de Aegorhinus superciliosus (G.) (Coleoptera: Curculionidae). Tesis Ingeniero Agrónomo. Universidad de La Frontera. (Temuco, Chile). 109 P.

SOLERVICENS, J. \& M. ELGUETA. 1994. Insectos de follaje de bosques pantanosos del norte chico, centro y sur de Chile. Rev. Chilena Ent. 21: 135-164.

ST. LEGAR, R. J.; A. K CHARNLEY \& R. M. COOPER. 1986. Cuticle-degrading enzymes of entomothogenic fungi: synthesis in culture on cuticle. J. Inverte. Pathol. 48: 85-95.

VÁSQUEZ, J. 1977. Antagonistas de larvas de Scarabaeidae presentes en las praderas de la provincia de Valdivia. Tesis Ingeniero Agrónomo. Universidad Austral de Chile. (Valdivia. Chile). 54 p. 
